\title{
Qiliqiangxin reduced cardiomyocytes apotosis and improved heart function in infarcted heart through Pink1/Parkin -mediated mitochondrial autophagy
}

Junyang Zhou ${ }^{1 \dagger}$, Zhixiao Wang ${ }^{2 \dagger}$, Yun He ${ }^{3}$, Xinxia Luo ${ }^{1}$, Wenjun Zhang ${ }^{3}, \mathrm{Li} \mathrm{Yu}^{1}$, Xiuying Chen ${ }^{4}, \mathrm{Xiju} \mathrm{He}^{1}$, Yahong Yuan', Xiaoli Wang ${ }^{1}$, Xinrong Guo ${ }^{1}$, Junming Tang ${ }^{1}$, Mingan Zhu', Dongsheng Li ${ }^{1}$ and Yan Ding ${ }^{1,5^{*}}$

\begin{abstract}
Background: Qiliqiangxin (QLQX) is a preparation refined from a traditional Chinese medicine compound. It plays an important role in protecting cardiac function after myocardial infarction (MI). However, the underline mechanism of QLQX action is not clear. The purpose of this study was to detect the effects of QLQX on mitophagy after MI.

Methods: Male FVB/NJ mice aged 8-10 weeks were underwent left coronary artery ligation and were orally administered either QLQX $(0.25 \mathrm{~g} / \mathrm{kg} / \mathrm{d})$ or saline. Twenty-eight days after surgical operation, the cardiac function of mice was detected by echocardiography. Electron Microscopy was used to observe the microstructure of cardiomyocytes. Myocardial apoptosis was examined by TdT-mediated dUTP Nick-End Labeling (TUNEL) and western blot. $\mathrm{H} 9 \mathrm{c} 2$ cells were cultured in a hypoxic incubator chamber $\left(5 \% \mathrm{CO}_{2}, 1 \% \mathrm{O}_{2}, 94 \% \mathrm{~N}_{2}\right)$ for $12 \mathrm{~h}$ and pretreated with or without QLQX $(0.5 \mathrm{mg} / \mathrm{mL})$. The cell apoptosis, reactive oxygen species (ROS), mitochondrial membrane potential and mitophagy were detected.

Results: When compared to sham group, the cardiac function of Ml mice decreased significantly, and their cardiomyocyte apoptosis and mitochondrial damage were more serious. These Ml-induced cardiac changes could be reversed by QLQX treatment. In vitro experiments also confirmed that QLQX could protect cardiomyocytes from hypoxia-induced apoptosis and mitochondrial damage. Further study indicated that QLQX could increase the expression of Pink1 and Parkin in cardiomyocytes.
\end{abstract}

Conclusion: Qiliqiangxin could reduce cardiomyocytes apotosis and improved heart function in infarcted heart through Pink1-mediated mitochondrial autophagy.

Keywords: Qiliqiangxin, Myocardial infarction, Mitophagy, Heart function

\footnotetext{
*Correspondence: dyywzx@163.com

†Junyang Zhou and Zhixiao Wang contributed equally to this work.

'Hubei Key Laboratory of Embryonic Stem Cell Research, Taihe Hospital, Hubei University of Medicine, Shiyan 442000, Hubei, China

${ }^{5}$ Biomedical Research Institute, Hubei University of Medicine, Taihe Hospital, Hubei University of Medicine, Shiyan 442000, Hubei, China

Full list of author information is available at the end of the article
}

(C) The Author(s). 2020 Open Access This article is licensed under a Creative Commons Attribution 4.0 International License, which permits use, sharing, adaptation, distribution and reproduction in any medium or format, as long as you give appropriate credit to the original author(s) and the source, provide a link to the Creative Commons licence, and indicate if changes were made. The images or other third party material in this article are included in the article's Creative Commons licence, unless indicated otherwise in a credit line to the material. If material is not included in the article's Creative Commons licence and your intended use is not permitted by statutory regulation or exceeds the permitted use, you will need to obtain permission directly from the copyright holder. To view a copy of this licence, visit http://creativecommons.org/licenses/by/4.0/ The Creative Commons Public Domain Dedication waiver (http://creativecommons.org/publicdomain/zero/1.0/) applies to the data made available in this article, unless otherwise stated in a credit line to the data. 


\section{Background}

The incidence and mortality of myocardial infarction are still high in most countries in the world [1]. Although great progress has been made in the treatment of myocardial infarction, such as early reperfusion therapy, which has significantly reduced the mortality rate of myocardial infarction patients $[2,3]$. However, at the same time, the morbidity and mortality of left ventricular remodeling and heart failure are increasing after myocardial infarction, which has become an increasingly worrying and challenging health problem $[4,5]$. At present, treatment strategies for preventing left ventricular remodeling after myocardial infarction are still limited. Therefore, it is very necessary to conduct in-depth research on the pathological mechanism of myocardial injury and ventricular remodeling after myocardial infarction, and to determine additional treatment targets and treatment schemes to prevent the adverse effects of left ventricular remodeling after myocardial infarction [6].

As a central organ of the circulatory system, the heart needs tremendous amounts of energy to maintain its dynamic function, while mitochondria in cardiomyocytes are fundamental providers of cardiac energy. Mitochondrial malfunction and structural abnormalities are bound to cause myocardial damage and lead to cardiac systolic and diastolic dysfunction [7]. Recent studies have shown that in most eukaryotic cells, autophagy can be induced by nutrient deficiency, oxidative stress, salt stress or infection to remove damaged organelles, such as mitochondria, or to degrade normal organelles to reduce the operational burden thereby maintaining cell survival [8]. Autophagy occurs at different times and intensities, which may have different effects on tissue cells in different ways. Moderate autophagy can regulate the intracellular environment and maintain the stability of the intracellular environment, while excessive autophagy may lead to autophagic cell death and cause tissue damage. Mitophagy is a kind of selective autophagy [9]. It has attracted more and more attention because of its close relationship with the quantity and physiological function of mitochondria. Mitochondria provide the main energy for myocardial contraction-relaxation and control the key survival and death pathways in cells. Therefore, it plays an important role in maintaining cardiac homeostasis.

Some natural medicines are very effective in certain diseases [10-16]. Qiliqiangxin (QLQX) is a compound preparation of 11 Chinese herbal medicines [17], it was approved by the China Food and Drug Administration in 2004 for the treatment of heart failure and was recommended as an ancillary drug in Chinese guidelines for diagnosis and treatment of heart failure. QLQX has been used in China for years to treat heart failure after MI. But there is a lack of scientific explanation on the mechanism of its effect. It has been reported QLQX could improves cardiac function and attenuates cardiac remodeling in rats with experimental myocardial infarction [18] .Recent studies have demonstrated the beneficial effects of QLQX on chronic heart failure [19], Shen $S^{\prime}$ study also indicated that QLQX attenuates adverse cardiac remodeling after MI in ovariectomized mice via activation of PPAR [20]. QLQX can also inhibit cardiomyocyte apoptosis on H9c2 cardiomyocytes [21].. Tao L also think QLQX warrants further investigation as a therapeutic intervention to mitigate remodeling and heart failure after AMI in mice [17]. Due to QLQX's complex composition, pharmacodynamic pathway and multiple targets, we need more experimental results in vitro and vivo to reveal the mechanism of QLQX to treat MI. In this study, a post-myocardial infarction heart failure model was used to evaluate the effect of QLQX on cardiac function and to explore the protective effect of mitosis on QLQX in cardiac remodeling.

\section{Methods}

Chemicals

QLQX was provided by Shijiazhuang Yiling Pharmaceutic (Hebei, China). The specific components of QLQX include Panax ginseng C. A. Mey. Astragalus membranaceus (Fisch.) Bunge. Aconitum carmichaeli Debx. Salvia miltiorrhiza Bge Descurainia sophia(L.)Webb. ex Prantl. Alisma orientalis (Sam.) Juzep. Cinnamomum cassia Presl Carthamus tinctorius L. Periploca sepium Bge. Citrus reticulata Blanco and Polygonatum odoratum (Mill.)Druce [22] and the labeled compounds have been certified and standardized according to the Chinese Pharmacopoeia (2010). In the current study, QLQX powder was prepared into $25 \mathrm{mg} / \mathrm{ml}$ solution with normal saline.

\section{Surgical operation}

Male FVB/NJ mice, aged 8 to10 weeks, were housed and used for experiments. All mice were purchased at the Institute of Biomedical Sciences of Nanjing University (Nanjing, China). Animal breeding, handling and surgical protocols were reviewed, approved and monitored by the Animal Care and Use Committee of Hubei University of Medicine. Briefly, after animals were induced deep anesthesia with $2 \%$ isoflurane, the heart was exposed by a left thoracotomy at the third intercostal space, and the anterior descending branch of the left coronary artery (LAD) is ligated with 6-0 suture at a position $2-3 \mathrm{~mm}$ below the left atrium. Then, the heart was placed back in situ, followed by evacuating of the air out of the thoracic cavity and closing the skin incision with a 4-0 nylon suture. The mice were placed on an electric blanket to recover and were closely observed. To relieve the pain in mice, one dose of buprenorphine $(0.1$ 
$\mathrm{mg} / \mathrm{kg}$ ) was given within $6 \mathrm{~h}$ after the operation, and another dose was given the next morning. For experiments, mice were intragastrically treated with QLQX $(0.25 \mathrm{~g} / \mathrm{kg} /$ d, QLQX group) or saline (saline group) for 4 weeks. Animals in the sham group were performed the same operation as the infarction group without ligation of the LAD.

\section{Echocardiography of heart}

The mice were anesthetized using $1.5 \%$ isoflurane and the Echocardiography was recorded with a Vevo1100 imaging system using a MS400 transducer. M-mode analysis was used to calculate ejection fraction, ventricle wall thickness, fractional shortening, and intra-ventricle diameter.

\section{Euthanasia}

Intraperitoneal injection of three times the anesthetic dose of $1 \%$ sodium pentobarbital solution.

\section{Immunohistochemistry analysis}

Immunohistochemistry was carried out as follows. In short, paraffin-embedded sections are stripped of paraffin, hydrated, and the antigen was repaired by microwave, then incubated in 3\% hydrogen peroxide for 10 min to remove endogenous peroxidase, and further incubated with goat serum for $10 \mathrm{~min}$ for blocking. Then, the tissue sections were incubated with Pink1 rabbit polyclonal antibody (Proteintech, 23,274-1-AP, 1:200) and Parkin rabbit polyclonal antibody (Proteintech, 14, 060-1-AP, 1:200) overnight at $4{ }^{\circ} \mathrm{C}$, followed with a horse radish peroxidase (HRP)-labeled goat anti-rabbit secondary antibody (ZSGB-BIO, PV-9001) for $20 \mathrm{~min}$ at room temperature. Next, the sections were incubated with 50ul diaminobenzidine (DAB) for 5-8 min and mounted with neutral gum. Using Olympus BX53 microscope to observe the staining and take photos.

\section{Assessment of myocardial infarct area}

Evan's blue and 2,3,5-triphenyltetrazolium chloride (TTC) double staining method was used to assess the size of myocardial infarction area [23]. About $0.2-0.3 \mathrm{ml}$ of $2 \%$ Evan's Blue dye solution was injected into the coronary arteries to identify non-ischemic areas. When the right side of the heart turned blue, the heart was rapidly peeled off and frozen at $-20^{\circ} \mathrm{C}$ for $30 \mathrm{~min}$. Then the heart was cut into 5 pieces with uniform thickness from apex to atrium, and incubated at $37^{\circ} \mathrm{C}$ for $15 \mathrm{~min}$ in $1 \%$ TTC $(0.1 \mathrm{~mol} / \mathrm{L} \mathrm{PBS})$ at $37^{\circ} \mathrm{C}$ for $15 \mathrm{~min}$. The infarct area (INF; white) and area at risk (AAR; red and white) of each segment were used to assess the myocardial infarct size.

\section{Electron microscopy}

Electron Microscopy examination was carried out according to the method of $\mathrm{He}$ et al. [24]. In short, 28 days after ligation of left anterior descending coronary artery, a small (about $1-\mathrm{mm}^{3}$ ) piece of tissue was taken out from the peripheral area of heart infarction, and repidly fixed with $2.5 \%$ glutaraldehyde for $2-4 \mathrm{~h}$, then fixed with $2 \% \mathrm{OsO}_{4}$ for $1 \mathrm{~h}$, and embedded in Acetone: 812 (Ladd Research). Then, the slides were double stained with Uranium and lead and observed and photographed with an HT7700 SS/FEI Tecnai G20 (Hitachi Limited).

\section{ROS and the mitochondrial membrane potential $(\Delta \Psi \mathrm{m})$ detection}

H9c2 cardiomyocytes were donated by Dr. He from Hubei university of medicine [17]. Cells were cultured in high glucose dulbecco's modified eagle medium (DMEM) supplemented with 10\% FBS (Gibco, C11995500), $100 \mathrm{IU} / \mathrm{mL}$ of penicillin and $100 \mu \mathrm{g} / \mathrm{mL}$ of streptomycin, and incubated in $95 \%$ air, $5 \% \mathrm{CO}_{2}$. The detection of reactive oxygen species (ROS) and $\Delta \Psi \mathrm{m}$ in H9c2 cells was carried out according to the specifications of ROS detection kit (Beyotime, S0033) and mitochondrial membrane potential detection kit (Solarbio, CA1310). For the determination of ROS, $1 \times 10^{5} /$ well $\mathrm{H} 9 \mathrm{c} 2$ cells were first cultured under normal conditions (control group), $\mathrm{O}_{2} 1 \%$ or $\mathrm{O}_{2} 1 \%+$ QLQX, and then incubated with reactive oxygen species sensitive dye $2^{\prime}, 7^{\prime}$ Dichlorodihydrofluorescein diacetate (DCFH-DA) solution at $37^{\circ} \mathrm{C}$ for $20 \mathrm{~min}$. In order to measure $\Delta \Psi \mathrm{m}$, cells were treated as described above and then incubated with $5,5^{\prime}, 6,6^{\prime}$ - Tetrachloro-1,1',3,3' -tetraethyl-imidacarbocyanine (JC-1) staining solution $(5 \mathrm{mg} / \mathrm{ml})$ at $37^{\circ} \mathrm{C}$ for 20 min. After staining, cells were washed twice with JC-1 staining buffer and detected by fluorescence microscope (Olympus FV3000RS). Fluorescence is measured at excitation/emission $485 / 580 \mathrm{~nm}$ (red) and then at excitation/ emission 485/530 nm (green). The results were analyzed with Image-Pro Plus software.

\section{Western blot}

Tissues or cells were lysed with protein lysate buffer radio immunoprecipitation assay (RIPA) (Beyotime, P00138). Supernatant of lysate was collected, and the protein concentrations were detected by bicinchoninic acid (BCA) method (Beyotime, P0012). The protein was isolated by $10 \%$ sodium dodecyl sulfate polyacrylamide gel electrophoresis (SDS-PAGE) and transferred to polyvinylidene fluoride (PVDF) membrane. The membrane was sealed with $5 \%$ skim milk solution at room temperature for $30 \mathrm{~min}$ and then incubated overnight at $4{ }^{\circ} \mathrm{C}$ with corresponding primary antibody, including Bcl-2 rabbit polyclonal antibody (Beyotime, AF0060, 1: 500), Bax rabbit monoclonal antibody (Beyotime, 
AF1270, 1:500), LC3 rabbit polyclonal antibody (protein technology, 18,725-1-AP, 1:500), Pink1 rabbit polyclonal antibody (protein technology, 23,274-1-AP, 1:500), Parkin rabbit polyclonal antibody. The next day, the membrane was washed with tris buffered saline tween (TBST) buffer and incubated with diluted horseradish peroxidase labeled goat anti-rabbit antibody (Beyotime, A0208, 1:1000) and anti-mouse antibody (Beyotime, A0216, 1:1000) at room temperature for $1 \mathrm{~h}$. The ultrasensitive chemiluminescence kit BeyoECL Star was used for color development, and the results were analyzed with image J 8.0.

\section{Analysis of mitophagic vacuoles}

H9c2 cells were transfected with green fluorescent protein-tagged microtubule-associated protein 1light chain 3 (Ad-GFP-LC3) and red fluorescent protein -tagged HBmTur-Mito and Ad-HBm (Tur-Mito-RFP, HANBIO), according to the manufacturer's instructions. The next day, cells treated with or without $1 \% \mathrm{O}_{2}$ or $1 \%$ $\mathrm{O}_{2}+$ QLQX for $12 \mathrm{~h}$. After the treatments, the cells were inspected with a fluorescence microscope (Olympus FV3000RS). The mitophagy was quantified by calculating the percentage of GFP-LC3 and Mito-RFP copositive autophagic vacuoles in cells.

\section{TUNEL assays}

Tissue slides and cells which were cultured with $1 \% \mathrm{O} 2$ or $1 \% \mathrm{O} 2+\mathrm{QLQX}$ for $12 \mathrm{~h}$, were reacted with terminal deoxynucleotidyl transferase (TdT) enzyme and $2^{\prime}$ Deoxyuridine $5^{\prime}$-Triphosphate (dUTP) at $37^{\circ} \mathrm{C}$ for $1 \mathrm{~h}$. Then 4', 6- diamino - 2- phenylindole (DAPI) (Beyotime, C1006) was used to stain the nucleus for $5 \mathrm{~min}$. Apoptosis was observed and photographed by fluorescence microscope (Olympus FV3000RS).

\section{Statistical analysis}

Prism 7.0 (Graph Pad) was used for statistical analysis of the data. All data were expressed as means \pm standard deviation (SD). The comparison of measurement data between two groups was carried out by independentsample $\mathrm{T}$ test. Survival curves were analyzed with the Kaplan-Meier tests. A $p$ value less than 0.05 was considered statistically significant.

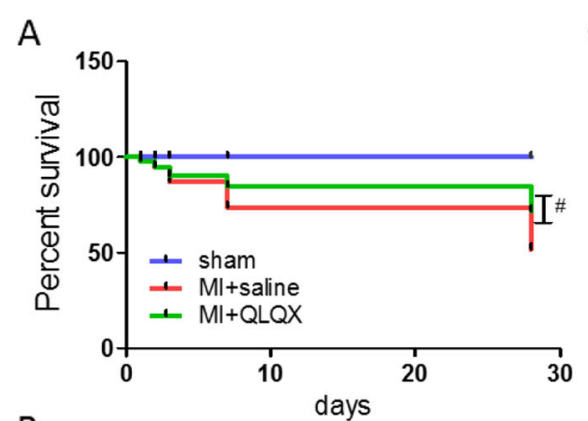

C

B

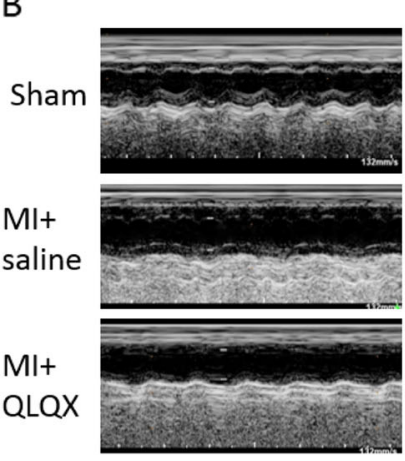

D

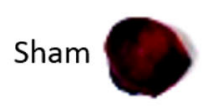

$\mathrm{MI}+$ saline

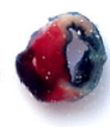

$\mathrm{Ml+}$

QLQX

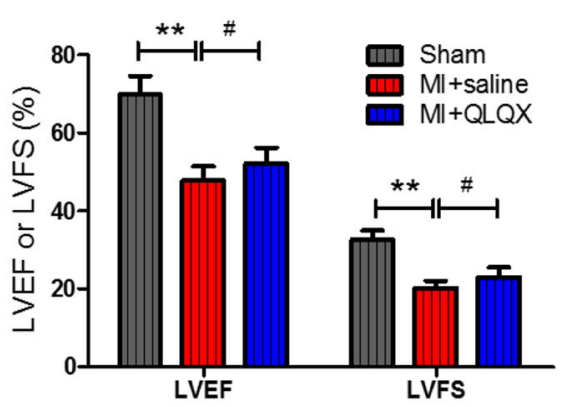

$\mathrm{E}$

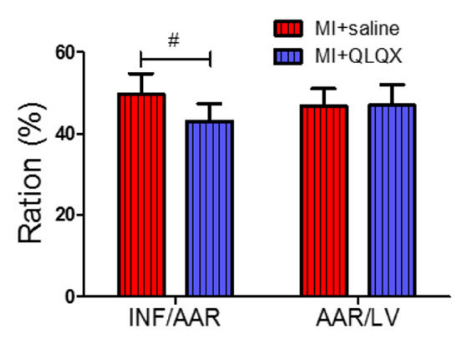

Fig. 1 Qiliqiangxin (QLQX) improves survival rate and cardiac function after myocardial infarction (MI). a: Survival rate. FVB/NJ mice were subjected to sham or Ml operation. The number of dead mice over a 4-wk period was counted daily. Survival curves were analyzed with the Kaplan-Meier tests. Data are presented as mean \pm SD, " ${ }^{\prime}$ compared to saline group, $P<0.05, n=10$ per group. $\mathbf{b}$ : Echocardiogram analysis. Representative echocardiographic images of FVB/NJ mice 4 weeks after MI surgery. c: QLQX improves cardiac function including preserving left ventricular fractional shortening (LVFS) and left ventricular ejection fraction (LVEF). Data are presented as mean $\pm \mathrm{SD},{ }^{* *}$ compared to Sham group, $P<0.01$, " compared to saline group, $P<0.05, n=8$ per group. $\mathbf{d}$ : Evan's blue/TTC stain. Heart tissue sections were stained with Evan's blue/TTC at 4 weeks after MI surgery. The infarct area (INF: white) and area at risk (AAR: red); blue is the normal myocardial blood supply area. e: The ratio of INF/AAR and AAR/LV. Data are presented as mean $\pm S D$, \# compared to saline group, $P<0.05, n=6$ per group. The comparison of measurement data between two groups was carried out by independent-sample $T$ test 


\section{Results}

QLQX improves survival rate and cardiac function after MI To investigate the effect of QLQX on MI, the survival rate of mice after MI operation was observed. Survival rates of saline group and QLQX-treated group were similar within the first $24 \mathrm{~h}$. Nevertheless, the mortality rate of saline group mortality was higher than QLQX group over 28 days (37.5\% for saline vs $22.5 \%$ for QLQX; $P<0.05$, Fig. 1a).

Echocardiogram was used to assess cardiac function of each group. Compared to sham controls, the contractile function of the infarcted anterior wall in both saline and QLQX groups were impaired from the 2-D M-mode imaging of the left ventricle. Furthermore, the cardiac function in the saline group was significantly worse than that in QLQX group (Fig. 1b). QLQX consistently improved cardiac functions including left ventricular ejection fraction (LVEF) and left ventricular shortening fraction (LVFS) (Fig. 1c, $P<0.05$ ). LVEF values were $69.87 \pm$ $4.68 \%, 47.84 \pm 3.56 \%$ and $52.16 \pm 4.15 \%$, and LVFS values were $32.62 \pm 2.33 \%, 20.22 \pm 1.8 \%$ and $22.92 \pm 2.56 \%$ for sham, saline and QLQX groups respectively.

The myocardial infarction size was measured by evan's blue/TTC double stain to determine the effects of QLQX on myocardial infarction. Representative myocardial infarct size images were shown in Fig. 1d. After 28 days of $\mathrm{MI}$, the infarct size of the saline group and the QLQX group increased significantly. Compared with saline group, the infarct area in QLQX group was significantly reduced (INF/AAR: $49.68 \pm 5.13 \%$ vs. $43.08 \pm 4.23 \%, P<0.05$, respectively. Figure $1 \mathrm{e})$. There was no significant difference between saline group and QLQX group in AAR/LV (AAR /LV: $46.78 \pm 24.34 \%$ vs. $47.13 \pm 5.01 \%$, respectively. $P>0.05$, Fig. $1 \mathrm{e})$.

\section{QLQX reduced cell apoptosis after myocardial infarction (MI) during remodeling phase}

Myocardial apoptosis is another characteristic of ventricular remodeling after myocardial infarction. The apoptotic cells were significantly decreased in the QLQX group as assessed by TUNEL staining when compared to saline group. The percentages of apoptotic cells were $4.03 \pm 0.58$, $22.33 \pm 1.76$ and $9.67 \pm 1.45$ for sham, saline and QLQX groups respectively (Fig. $2 \mathrm{a} \& \mathrm{~B}, P<0.01$ ). The expression levels of Bax and Bcl-2 were detected by Western blot (Fig. 2c). The ratio of $\mathrm{Bax} / \mathrm{Bcl}-2$ was used to measure the apoptosis of cardiomyocytes and it was significantly increased after MI. However, QLQX could obviously reverse this phenomenon (Fig. 2d). The ratios of $\mathrm{Bax} / \mathrm{Bcl}-2$ in

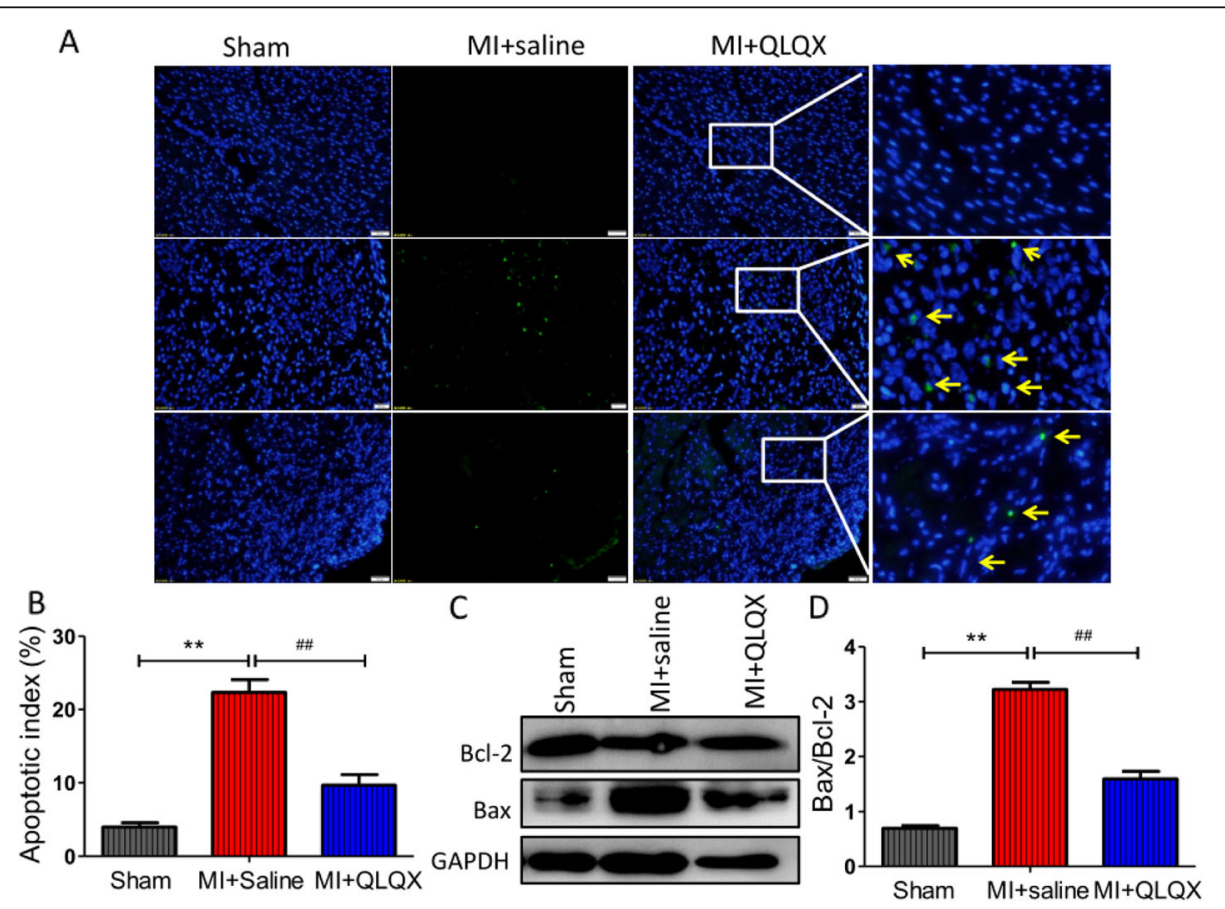

Fig. 2 Qiliqiangxin (QLQX) reduces cardiomyocyte apoptosis after myocardial infarction (MI) during remodeling phase. a: TUNEL Assays detected the apoptosis cells in the infarction peripheral tissues. The yellow arrow indicated the apoptosis cells. $\mathbf{b}$ : Column diagram showed the percentage of apoptosis cells, data are presented as mean $\pm \mathrm{SD},{ }^{* *}$ Compared to sham group, $P<0.01$; ${ }^{\# \#}$ Compared to saline group, $P<0.01, n=4$ per group. c: Western blot detected the apoptosis marker Bax and $\mathrm{BCl}-2$. d: Column diagram showed the ratio of Bax/BCl-2. Data are presented as mean \pm $\mathrm{SD},{ }^{* *}$ Compared to sham group, $P<0.01$; \#\# Compared to saline group, $P<0.01, n=4$ per group. The comparison of measurement data between two groups was carried out by independent-sample $T$ test 
sham, saline and QLQX groups were $0.7 \pm 0.05,3.22 \pm$ 0.13 and $1.58 \pm 0.14$ respectively $(P<0.01)$.

\section{QLQX increased mitophagy after MI surgery}

To further detect the underline mechanism, transmission electron microscopy (TEM) was used to observe the mitochondrial structure. The results showed that on day 28 the mitochondrial structure was preserved and orderly arranged, and there were no significant structural variations in sham group. In saline group, mitochondria were aggregated at the myofilament breaks, and some of them were evidently vacuolated. The mitochondria of QLQX group were arranged more regularly than that of saline group, and some of them were filled in the area of myofibril breakage and loss (Fig. 3a). The percentage of mitochongria incorporated by autophagosome of QLQX group was significantly higher than the saline group (Fig. 3b). Further study indicated that QLQX treatment could repress the decrease of Pink 1 , Parkin and pParkin induced by MI, which was demonstrated in the immunohistochemistry analysis (Fig. 3c). The positive area of Pink1, Parkin and pParkin in saline group was significantly decreased when compared to sham group, but QLQX treatment could suppress this phenomenon. The Pink1 positive areas were $58.67 \pm 5.84,18.68 \pm 1.8$ and
$74.87 \pm 7.03$ in sham, saline and QLQX groups respectively (Fig. $3 \mathrm{~d}, P<0.01$ )., the Parkin positive areas were $38.67 \pm 4.03,22.78 \pm 2.34$ and $39.13 \pm 4.18$ in sham, saline and QLQX groups respectively (Fig. 3d, $P<0.01$ ), the pParkin positive areas were $35.78 \pm 3.35,20.56 \pm 1.63$ and $28.65 \pm 2.97$ in sham, saline and QLQX groups respectively (Fig. $3 \mathrm{~d}, P<0.05$ ).

\section{Effect of QLQX on hypoxic injury in H9c2 cardiac cells}

We assessed the apoptosis in different group with TUNEL assay. The viability of H9c2 cardiac cells following hypoxia treatment was shown in Fig. 4a. The percentage of apoptosis cell after hypoxia treatment was markedly increased $(2.07 \pm 0.41 \%$ vs. $15.36 \pm 2.8 \%$ for control vs. $\left.1 \% \mathrm{O}_{2}, P<0.01\right)$. This effect was significantly alleviated by QLQX treatment (QLQX: $8.25 \pm 0.87$ vs. $15.36 \pm 1.8 \%$ for $1 \% \mathrm{O}_{2}+\mathrm{QLQX}$ vs. $1 \% \mathrm{O}_{2}, P<0.01$, Fig. $4 \mathrm{~b}$ ). The results of western blot revealed that compared to saline group, the expression of pro-apoptotic molecule Bax was decreased, while the anti-apoptotic molecule B-cell lymphoma 2 (Bcl-2) was increased in QLQX-treated mice after MI (Fig. 4c\&d). Collectively, these datas confirmed that QLQX attenuates hypoxiainduced H9c2 cardiomyocyte apoptosis.
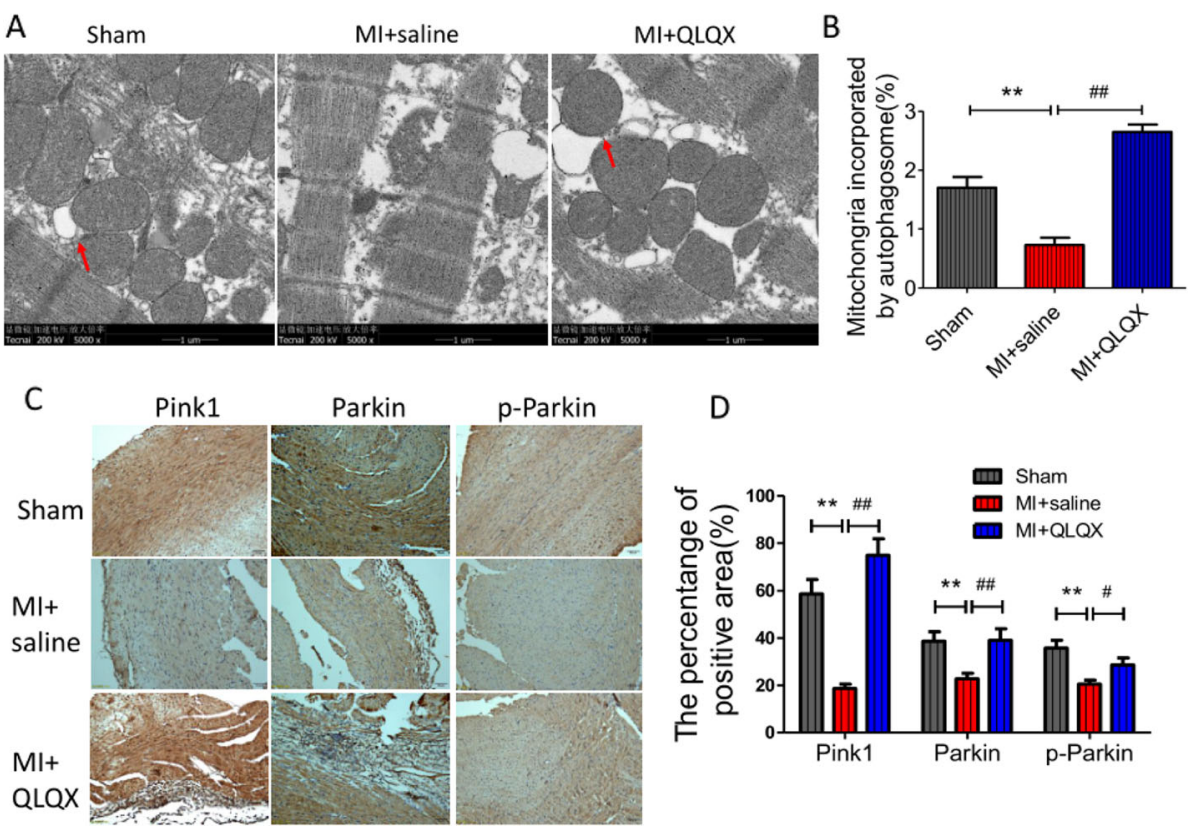

D

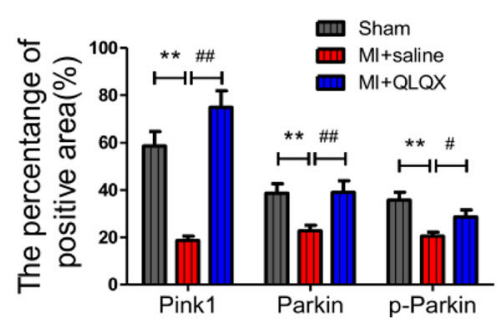

Fig. 3 QLQX increases mitophagy after MI surgery. a: Transmission electron microscopy (5000x) detected mitochondrial structure and autophagy in infarction peripheral tissues, the red arrows indicated the autophagosome. $\mathbf{b}$ : Column diagram showed the percentage of mitochongria incorporated by autophagosome, data are presented as mean $\pm \mathrm{SD},{ }^{* *}$ Compared to sham group, $P<0.01$; ${ }^{\# \# ~ C o m p a r e d ~ t o ~ s a l i n e ~ g r o u p, ~} P<0.01$, $n=3$ per group. $\mathbf{c} \& \mathbf{d}$ : Immunochemical staining detected the expression of Pink1, Parkin and p-Parkin in the heart at 4 weeks after MI. Representative images were selected from 3 separate experiments. Data are presented as mean $\pm \mathrm{SD},{ }^{* *}$ Compared to sham group, $P<0.01 ;{ }^{\# \#}$ Compared to saline group, $P<0.01$, " Compared to saline group, $P<0.05, n=4$ per group. The comparison of measurement data between two groups was carried out by independent-sample T test 


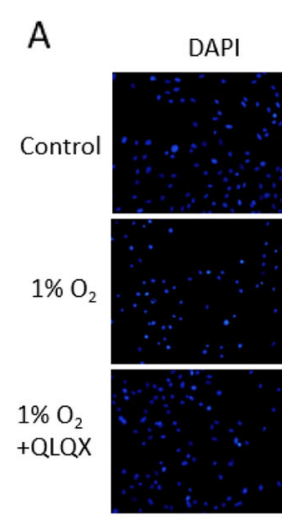

C

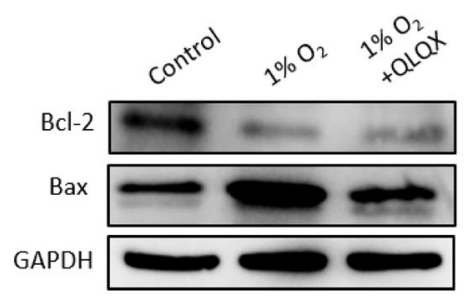

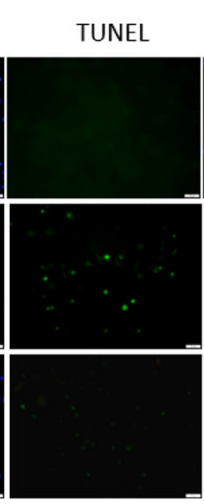

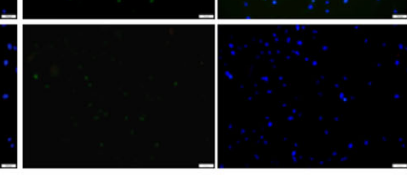

D
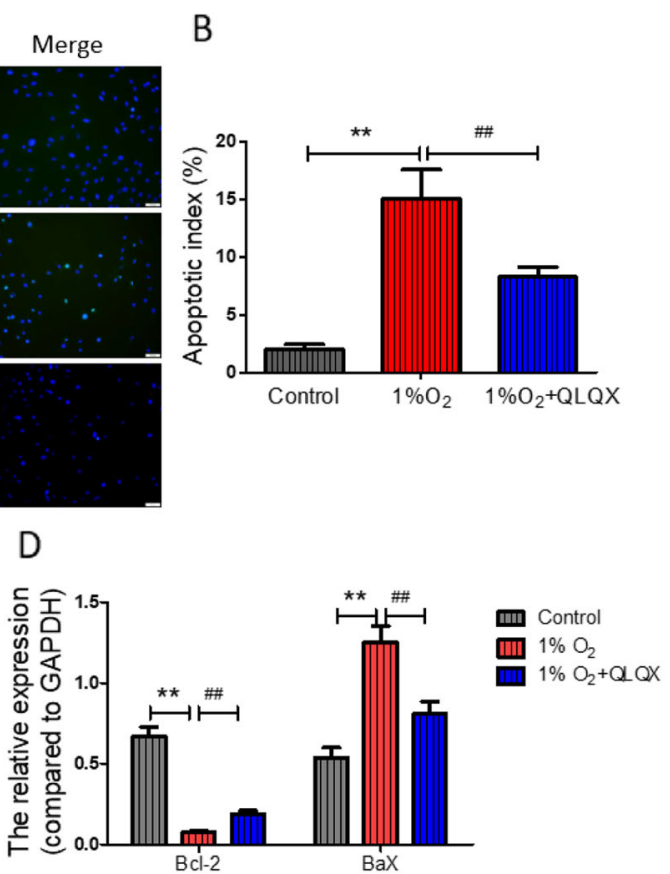

Fig. 4 Effect of QLQX on hypoxic injury in H9c2 cardiac cells. a: TUNEL staining was used to detect the apoptosis of H9c2 cells treated with 1\% $\mathrm{O}_{2}$ or $1 \% \mathrm{O}_{2}+$ QLQX for $12 \mathrm{~h}$, the green fluorescence indicated the apoptotic cells. $\mathbf{b}$ : Column diagram showed percentage of apoptosis cells. Data are presented as mean $\pm \mathrm{SD},{ }^{*}$ Compared to control group, $P<0.01$; ${ }^{\# \#}$ Compared to $1 \% \mathrm{O}_{2}$ group, $P<0.01 . n=3$ per group. c: Western blot analysis of $\mathrm{Bax}$ and $\mathrm{BCl}-2$ levels in $\mathrm{H} 9 \mathrm{c} 2$ cells. $\mathbf{d}$ : Column diagram showed the relative expression levels of Bax and Bcl-2. The statistical data are presented as mean \pm SD of three independent experiments. Data are presented as mean \pm SD, ${ }^{* *}$ Compared to control group, $P<0.01$; \#\# Compared to $1 \% \mathrm{O}_{2}$ group, $P<0.01 . n=3$ per group. The comparison of measurement data between two groups was carried out by independent-sample T test

\section{QLQX reduced ROS production and increased $\Delta \Psi \mathrm{m}$}

The change of ROS generation was detected by using the DCFH-DA dye. Hypoxia would increase the staining of DCFH-DA, whereas the QLQX treatment could reduce the staining of DCFH-DA induced by hypoxia (Fig. 5a). The differences of ROS production between the three groups were statistically significant. Compared to control group $(9.67 \pm 1.2)$, the percentage of green fluorescence positive cells in hypoxia group significantly increased, which was $20.5 \pm 2.1 \quad(P<0.01)$. However, QLQX treatment clearly alleviated this hypoxia-induced cell apoptosis $(16 \pm 3.1, P<0.01$, Fig. $5 b)$. To investigate the mitochondrial function, the mitochondrial potential $(\Delta \Psi \mathrm{m})$ was measured and showed a dramatical decrease in hypoxia group (Fig. 5c). However, QLQX treatment restored the $\Delta \Psi \mathrm{m}$, the ratios of red to green fluorescence positive cells were $5.6 \pm 0.61,1 \pm 0.15$ and $3.2 \pm 0.3$ for control, $1 \% \mathrm{O}_{2}$ and $1 \% \mathrm{O}_{2}+$ QLQX group respectively $(P<0.01$, Fig. $5 d)$.

\section{QLQX increased mitophagy level in hypoxic injured H9c2 cells}

To detect the influence of QLQX on the process of mitophagy in hypoxic injured H9c2 cells, we transfected
$\mathrm{H} 9 \mathrm{c} 2$ cells with adenovirus that stably expresses the GFP-tagged LC3 and RFP-tagged HBmTur-Mito, which can co-localize autophagosome and mitochondria. As shown in Fig. 6a, compared to control group, the number of green fluorescent protein (GFP) and red fluorescent protein (RFP) co-positive dots in $1 \% \mathrm{O}_{2}$ group were significantly decreased. However, when treated with QLQX, the number of autophagosome was increased. The mitochondrial/LC3 co-localization coefficient in control, $1 \% \mathrm{O}_{2}$ and QLQX groups were $39.25 \pm 3.19$, $12 \pm 2.04,29.5 \pm 1.56$ respectively (Fig. $6 \mathrm{~b}, \quad P<0.05$ ). Compared to saline group, western blot results also indicated that the level of Pink1, Parkin and pParkin were increased in the QLQX treatment group (Fig. 6c\&d). These results showed that QLQX treatment could increase the mitophagy level in $\mathrm{H} 9 \mathrm{c} 2$ cells.

\section{Discussion}

Myocardial infarction (MI) is an irreversible death of myocardial cells in the corresponding area of coronary artery occlusion. The high incidence and younger age of myocardial infarction have become more and more severe. The World Health Organization (WHO) estimates that about 23 million people accounting for $12.8 \%$ of 


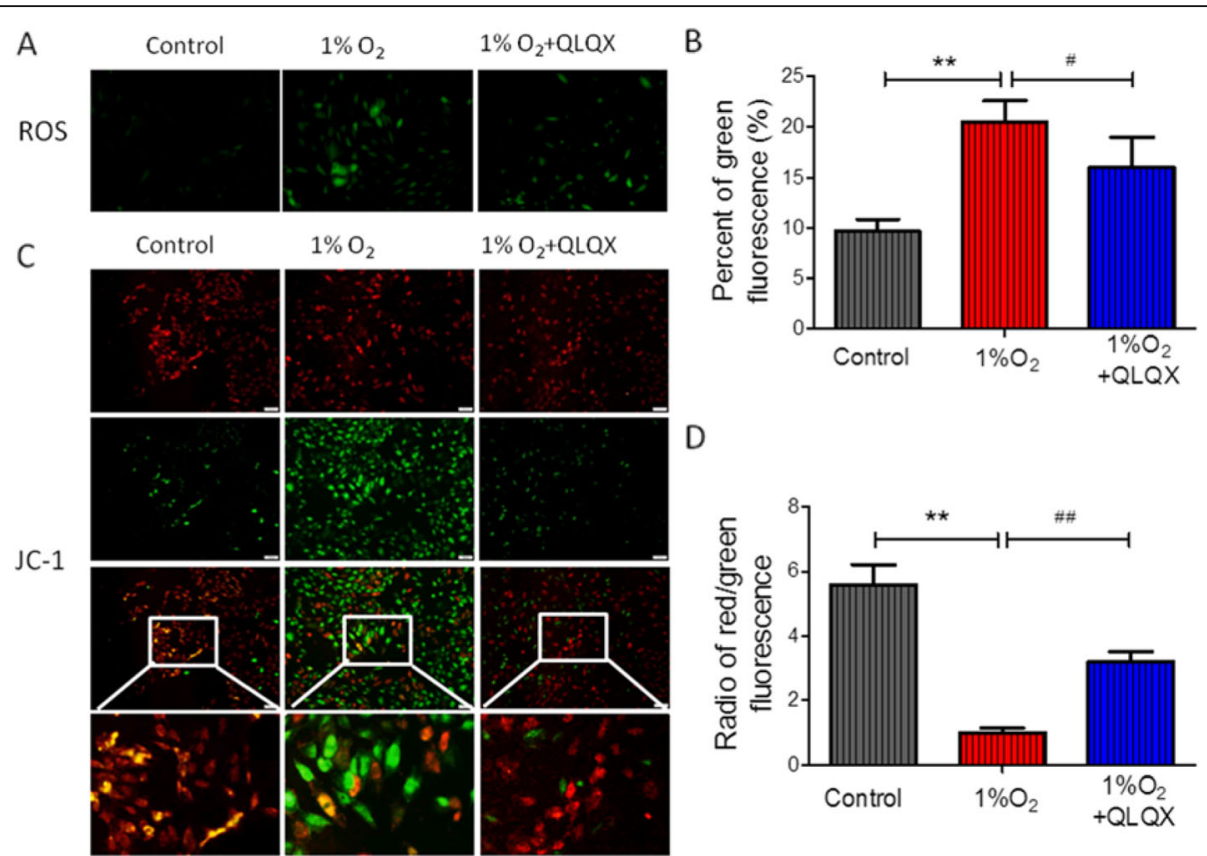

Fig. 5 QLQX reduced ROS production and increased $\triangle \Psi m$. a: Representative images of ROS staining. b: Quantifications of ROS fluorescence intensity in different groups. Data are presented as mean $\pm \mathrm{SD}_{1}{ }^{* *}$ Compared to control group, $P<0.01$; \#\# Compared to $1 \% \mathrm{O}_{2}$ group, $P<0.01$. $n=3$ per group. c: Mitochondrial membrane potential $\left(\Delta \Psi_{\mathrm{m}}\right)$ detected by JC-1 staining. Red fluorescence represents the mitochondrial aggregate form of JC-1, indicating an intact mitochondrial membrane potential. Green fluorescence represents the monomeric form of JC-1, indicating the dissipation of $\Delta \psi \mathrm{m}$. d: Ratio of the red/green fluorescence intensity. Data are presented as mean $\pm \mathrm{SD},{ }^{* *}$ Compared to control group, $P<0.01$; ${ }^{\# \#}$ Compared to $1 \% \mathrm{O}_{2}$ group, $P<0.01 . n=3$ per group. The comparison of measurement data between two groups was carried out by independent-sample $T$ test

total deaths will die of cardiovascular disease each year by 2030 [25]. The damage of heart function after myocardial infarction and the consequent decline in quality of life have also become increasingly serious problems in public health care [26]. Therefore, how to protect myocardium and improve cardiac function as much as possible after myocardial infarction is an urgent challenge for clinicians.

Cardiomyocyte apoptosis is vital to the pathological process of heart failure. Cardiomyocytes lost through apoptosis are not renewable, thus inhibiting cardiomyocyte apoptosis may have great influence to the therapeutic of heart failure. QLQX is a Chinese patent medicine developed according to the theory of collateral disease. It has exhibited remarkable curative effect in clinical applications and holds the traditional functions of strengthening heart, diuresis and vasodilation [17, 19, 20, 24]. Basic research shows that QLQX could restrain hypoxia-induced injury in primary rat cardiac microvascular endothelial cells via promoting glycolysis in a HIF-1a-dependent manner [27]. During hypoxia, cardiac microvascular endothelial cells (CMVECs) promote the expression of vascular endothelial growth factor (VEGF) and QLQX could attenuate this injury via facilitating hypoxia inducible factor- $1 \alpha(\mathrm{HIF}-1 \alpha)$ dependent glycolysis [28]. However, the mechanism of QLQX on apoptosis still remains unknown.
In the present study, we established mice myocardial infarction model by ligation of left coronary artery [29]. The results showed that QLQX could significantly reduce the infarct area, improve the heart function and survival rate of mice at 28 days after surgery. $\mathrm{Bcl}-2$-related protein family is the main regulator of cell apoptosis signal transduction pathway, and the ratio of anti-apoptosis protein $\mathrm{Bcl}-2$ to pro-apoptosis protein Bax determines whether cells undergo apoptosis when stimulated by apoptosis signal $[28,30]$. Our study found that after ligation of left coronary artery, the expression of Bax increased significantly while the expression of Bcl-2 decreased significantly in $\mathrm{MI}+$ saline group compare to sham group. However, QLQX could obviously restore the imbalance of Bcl-2/ Bax. This result indicated that QLQX plays an antiapoptosis protective role on myocardial cells. Apoptosis is a highly programmed cell death. Cardiomyocyte apoptosis contains cell surface death receptor signaling pathway and intracellular mitochondrial pathway two classical signaling pathways. The cell surface death receptor signaling pathway is mainly stimulated by Fas ligand or ligand of death receptor, such as tumor necrosis factor $\alpha$ (TNG- $\alpha$ ), while mitochondrial pathway can be activated by myocardial hypoxia, ischemia, oxidative stress, anticancer drugs, deoxyribonucleic acid (DNA) damage and other stimuli [31]. 

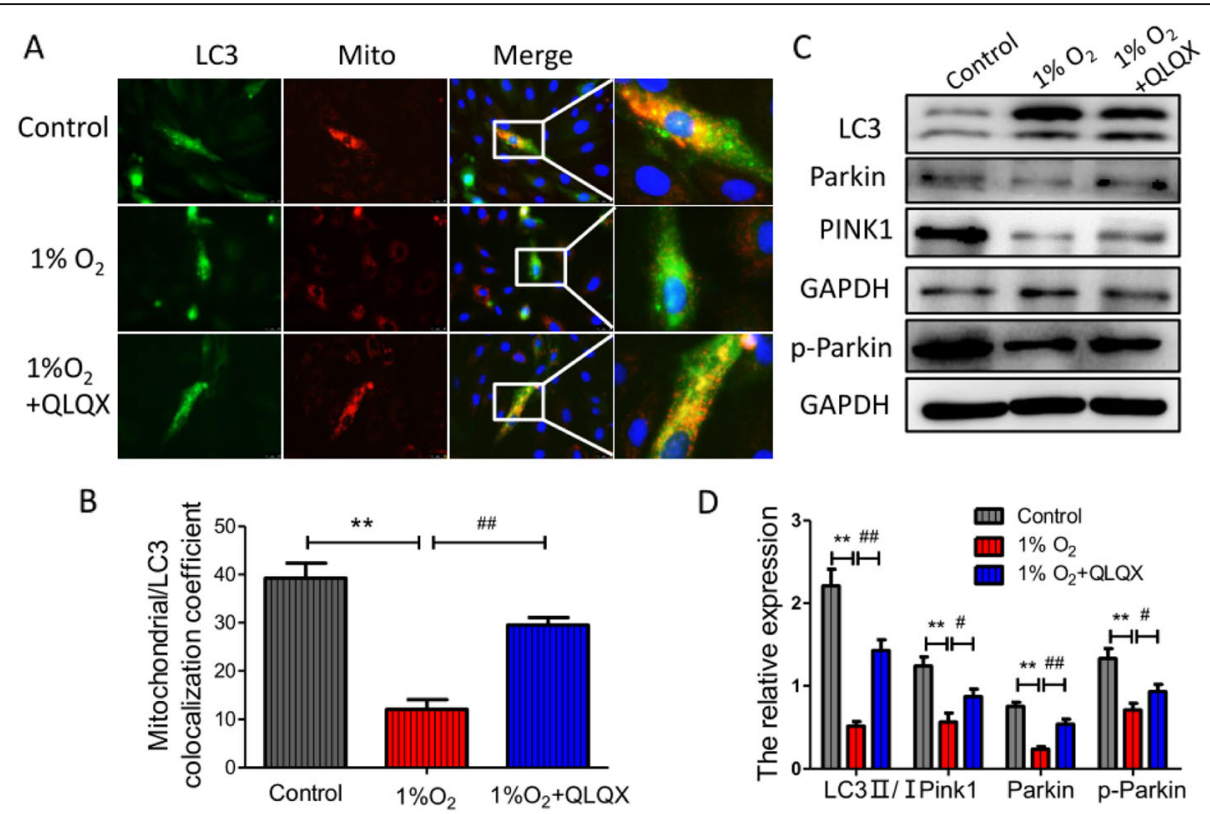

Fig. 6 QLQX increased mitophagy level in hypoxic injured H9c2 cells. a: Representative photomicrographs of GFP-LC3 and red fluorescent protein (RFP)-tagged HBmTur-Mito staining in $\mathrm{H} 9 \mathrm{c} 2$ cells. The green fluorescence dots indicate the autophagy. The red fluorescence dots indicate the mitochondria. The orange fluorescence indicates the mitophagy, 400X. b: The co-localization efficient of Mitochondria and LC3. Data are presented as mean $\pm \mathrm{SD}{ }^{* *}$ Compared to control group, $P<0.01 ;{ }^{\# \#}$ Compared to $1 \% \mathrm{O}_{2}$ group, $P<0.01 . n=3$ per group. $\mathbf{c}$ : Western blot analysis of mitophagy markers of LC3, Parkin, Pink1and p-Parkin in different groups. $\mathbf{d}$ : Column diagram showing the relative expression level of $L C 3$, Pink1,Parkin and pParkin. Data are presented as mean $\pm \mathrm{SD},{ }^{* *}$ Compared to control group, $P<0.01$; $\# \#$ Compared to $1 \% \mathrm{O}_{2}$ group, $P<0.01$, ${ }^{\#}$ Compared to $1 \% \mathrm{O}_{2}$ group, $P<0.05, n=3$ per group. The comparison of measurement data between two groups was carried out by independent-sample T test

Mitochondria are important organelles widely found in eukaryotic cells. It is the main site for cells to oxidize and produce adenosine-triphosphate (ATP), providing 95\% of the energy needed for cell life activities, known as the "cell power plant". The structure and biochemical functions of mitochondria are complex, sensitive and changeable. Changes of intracellular and extracellular factors can cause structural abnormality and malfunction of mitochondria [32, 33]. Therefore, mitochondria are often used as indicators for the changes of microenvironmental factors and even for the diagnosis of certain disease [34]. Mitophagy, as a selective autophagy, is a process of removing damaged or excessive mitochondria in cells [35], which refers to the process of depolarization damage of mitochondria in cells under the stimulation of ischemia, hypoxia, oxidative stress and aging, thus activating autophagy-lysosome pathway, completing the degradation of damaged mitochondria and maintaining the homeostasis of cell environment. Mitophagy could timely remove damaged or excessive mitochondria thereby maintaining the normal function of the body cells. In this study, by using transmission electron microscopy, we found that after MI, the autophagy level of left ventricular decreased significantly, while QLQX significantly increased the level of myocardial autophagy after MI. In vitro $\mathrm{H} 9 \mathrm{c} 2$ cell assays also showed that QLQX treatment could significantly improve mitochondrial dysfunction caused by hypoxia, presumably through reducing ROS production and the level of mitochondrial membrane potential. QLQX treatment also facilitated the mitochondrial/LC3 co-localization. These results indicated that QLQX could significantly improve myocardial injury and increase mitophagy level after MI.

Mitophagy includes four stages: earlier stage, early stage, middle stage and late stage. Each stage has different characteristics. The proteins involved in this process mainly includes: protein encoding mitochondrial outer membrane kinase (Pink1), E3 ubiquitin ligase (Parkin), B cell lymphoma/ leukemia-2 (Bcl-2), adenovirus interaction protein 3 (BNIP-3), autophagy-related gene (ATG) protein and Uth1 protein. Pink1 and Parkin proteins can induce mitophagy, remove excessive or damaged mitochondria, and maintain the stability of mitochondrial structure and function. The BNIP3 protein located in the outer membrane of mitochondria plays a significant role in the clearance of mitophagy in the process of mitochondrial development. ATG protein system is mainly involved in the formation of early mitophagy. Uth1 protein is a protein located in mitochondria. Mutation or deletion of Uthl will cause cells to be unable to remove damaged or aging mitochondria [35-37]. 
In our experiment, the data showed that Pink1, Parkin and pParkin levels decreased after MI, while QLQX could improve this phenomenon. This point was also confirmed in our in vitro cell experiments.

\section{Conclusions}

Taken together, our results indicated for the first time that QLQX could obviously improve the survival rate and the heart function of mice after MI presumably through Pink 1/Parkin signaling pathway-mediated increase of mitophagy and could reduce cardiomyocytes apoptosis. This study will provide new supportive evidence for the clinical application of QLQX.

\section{Supplementary information}

Supplementary information accompanies this paper at https://doi.org/10. 1186/s12906-020-02992-7.

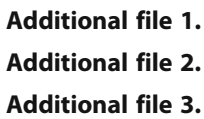

\section{Abbreviations}

QLQX: Qiliqiangxin; MI: Myocardial infarction; TUNEL: TdT-mediated dUTP Nick-End Labeling; DMEM: Dulbecco's modified eagle medium; ROS: Reactive oxygen species; LAD: Left coronary artery; DAB: Diaminobenzidine; HRP: Horse radish peroxidase; TTC: 2,3,5-triphenyltetrazolium chloride; INF: Infarct area; AAR: Area at risk; DCFH-DA: 2',7'-Dichlorodihydrofluorescein diacetate; JC-1: 5,5',6,6'-Tetrachloro-1,1',3,3'-tetraethyl-imidacarbocyanine; RIPA: Radio immunoprecipitation assay; BCA: Bicinchoninic acid; SDSPAGE: Sodium dodecyl sulfate polyacrylamide gel electrophoresis; PVDF: Polyvinylidene fluoride; TBST: Tris buffered saline tween; Ad-GFPLC3: Green fluorescent protein-tagged microtubule-associated protein 1 light chain 3; Tur-Mito-RFP, HANBIO: Red fluorescent protein -tagged HBmTur-Mito and Ad-HBm; TdT: Terminal deoxynucleotidyl transferase; DAPI: 4', 6- diamino - 2- phenylindole; dUTP: 2'-Deoxyuridine 5'-

Triphosphate; SD: Standard deviation; LVEF: Left ventricular ejection fraction; LVFS: Left ventricular shortening fraction; TEM: Transmission electron microscopy; GFP: Green fluorescent protein; RFP: Red fluorescent protein; WHO: World Health Organization; CMVECs: Cardiac microvascular endothelial cells; VEGF: Vascular endothelial growth factor; HIF-1a: Hypoxia inducible factor-1a; TNG-a: Tumor necrosis factor a; DNA: Deoxyribonucleic acid; ATP: Adenosine-triphosphate; ATG: Autophagy-related gene; Pink1: Protein encoding mitochondrial outer membrane kinase; Parkin: E3 ubiquitin ligase; Bcl-2: B cell lymphoma/ leukemia-2; BNIP-3: Adenovirus interaction protein 3

\section{Acknowledgements}

Not applicable.

\section{Authors' contributions}

$\mathrm{JZ}, \mathrm{ZW}$ and $\mathrm{XL}$ contributed to the construction of myocardial infarction model; $Y H$ and $W Z$ contributed to the cardiac echocardiography; $L Y, X C, Y Y$, $\mathrm{XH}$ contributed to immunohistochemistry and molecular biology experiments; XW, XG,JT contributed to cell experiments, MZ, DL and YD contributed to the manuscript writing. The author(s) read and approved the final manuscript.

\section{Funding}

The present study was supported by the Free Exploration Project of Hubei University of Medicine (FDFR201803), the Talent Start -up Fund of Hubei University of Medicine (2017QDJZR02), funder Yan Ding contributed to the manuscript writing. Guangxi Zhuang Autonomous Natural Science Foundation (2018JJA140751), funder Zhixiao Wang contributed to the construction of myocardial infarction model. The Hubei Provincial Technology Innovation Project (2016ACA157), funder Dongsheng L contributed to the manuscript writing. Principal Investigator Grant of Hubei
University of Medicine (HBMUPI201803), funder Xinrong Guo contributed to cell experiments.

Knowledge Innovation Program of Science and Technology Department of Hubei Province(K1223717), The National Natural Science Foundation of China (81700769, funder Junming Tang contributed to the manuscript writing. Collection, analysis and writing of this project were financially supported by resources of t Hubei University of Medicine, National Natural Science Foundation, Guangxi Science and Technology Department, Hubei Science and Technology Department.

Availability of data and materials

All data is contained within the manuscript and additional files.

Ethics approval and consent to participate

Animal breeding, handling and surgical protocols were reviewed, approved and monitored by the Animal Care and Use Committee of Hubei University of Medicine.

Consent for publication

Not applicable in this section.

\section{Competing interests}

The authors declare that they have no competing interest.

\section{Author details}

${ }^{1}$ Hubei Key Laboratory of Embryonic Stem Cell Research, Taihe Hospital, Hubei University of Medicine, Shiyan 442000, Hubei, China. ${ }^{2}$ Cardiovascular Department, Hubei University of Medicine, Taihe Hospital, Hubei University of Medicine, Shiyan 442000, Hubei, China. ${ }^{3}$ Ultrasonography Department, Hubei University of Medicine, Taihe Hospital, Hubei University of Medicine, Shiyan 442000, Hubei, China. ${ }^{4}$ Laboratory Department of the First Affiliated Hospital of Guizhou Medical University, Guiyang 550000, Guizhou, China. ${ }^{5}$ Biomedical Research Institute, Hubei University of Medicine, Taihe Hospital, Hubei University of Medicine, Shiyan 442000, Hubei, China.

Received: 8 August 2019 Accepted: 16 June 2020

Published online: 02 July 2020

\section{References}

1. Heusch G, Libby P, Gersh B, Yellon D, Bohm M, Lopaschuk G, Opie L. Cardiovascular remodelling in coronary artery disease and heart failure. Lancet. 2014;383:1933-43.

2. Seropian IM, Toldo S, Van Tassell BW, Abbate A. Anti-inflammatory strategies for ventricular remodeling following ST-segment elevation acute myocardial infarction. J Am Coll Cardiol. 2014;63:1593-603.

3. Mezzaroma E, Toldo S, Farkas D, Seropian IM, Van Tassell BW, Salloum FN, Kannan HR, Menna AC, Voelkel NF, Abbate A. The inflammasome promotes adverse cardiac remodeling following acute myocardial infarction in the mouse. Proc Natl Acad Sci U S A. 2011;108:19725-30.

4. Devaux Y, Vausort M, McCann GP, Zangrando J, Kelly D, Razvi N, Zhang L, $\mathrm{Ng}$ LL, Wagner DR, Squire IB. MicroRNA-150: a novel marker of left ventricular remodeling after acute myocardial infarction. Circ Cardiovasc Genet. 2013;6:290-8.

5. Vilahur G, Juan-Babot O, Pena E, Onate B, Casani L, Badimon L. Molecular and cellular mechanisms involved in cardiac remodeling after acute myocardial infarction. J Mol Cell Cardiol. 2011;50:522-33.

6. Bulluck H, Frohlich GM, Nicholas JM, Mohdnazri S, Gamma R, Davies J, Sirker A, Mathur A, Blackman D, Garg P, et al. Mineralocorticoid receptor antagonist pre-treatment and early post-treatment to minimize reperfusion injury after ST-elevation myocardial infarction: the MINIMIZE STEMI trial. Am Heart J. 2019;211:60-7.

7. Tahrir FG, Langford D, Amini S, Mohseni AT, Khalili K. Mitochondrial quality control in cardiac cells: Mechanisms and role in cardiac cell injury and disease. J Cell Physiol. 2019:234:8122-33.

8. Saha S, Panigrahi DP, Patil S, Bhutia SK. Autophagy in health and disease: A comprehensive review. Biomed Pharmacother. 2018:104:485-95.

9. Mizushima N, Komatsu M. Autophagy: renovation of cells and tissues. Cell. 2011:147:728-41.

10. Bishayee A, Block K. A broad-spectrum integrative design for cancer prevention and therapy: The challenge ahead. Semin Cancer Biol. 2015; 35(Suppl):S1-4. 
11. Al-Dabbagh B, Elhaty IA, Al HA, Al SR, El-Awady R, Ashraf SS, Amin A. Antioxidant and anticancer activities of Trigonella foenum-graecum, Cassia acutifolia and Rhazya stricta. BMC Complement Altern Med. 2018;18:240.

12. Amin A, Bajbouj K, Koch A, Gandesiri M, Schneider-Stock R. Defective autophagosome formation in p53-null colorectal cancer reinforces crocininduced apoptosis. Int J Mol Sci. 2015;16:1544-61.

13. Hamza AA, Ahmed MM, Elwey HM, Amin A. Melissa officinalis Protects against Doxorubicin-Induced Cardiotoxicity in Rats and Potentiates Its Anticancer Activity on MCF-7 Cells. PLoS One. 2016;11:e167049.

14. Ashktorab H, Soleimani A, Singh G, Amr A, Tabtabaei S, Latella G, Stein U, Akhondzadeh S, Solanki N, Gondre-Lewis MC, et al. Saffron: The Golden Spice with Therapeutic Properties on Digestive Diseases. Nutrients. 2019;1 1:943.

15. Amin A, Hamza AA, Daoud S, Khazanehdari K, Hrout AA, Baig B, Chaiboonchoe A, Adrian TE, Zaki N, Salehi-Ashtiani K. Saffron-based Crocin prevents early lesions of liver Cancer: in vivo, in vitro and network analyses. Recent Pat Anticancer Drug Discov. 2016;11:121-33.

16. Al-Hrout A, Chaiboonchoe A, Khraiwesh B, Murali C, Baig B, El-Awady R, Tarazi H, Alzahmi A, Nelson DR, Greish YE, et al. Safranal induces DNA double-strand breakage and ER-stress-mediated cell death in hepatocellular carcinoma cells. Sci Rep. 2018;8:16951.

17. Tao L, Shen S, Fu S, Fang H, Wang X, Das S, Sluijter JP, Rosenzweig A, Zhou $Y$, Kong $X$, et al. Traditional Chinese medication Qiliqiangxin attenuates cardiac remodeling after acute myocardial infarction in mice. Sci Rep. 2015; 5:8374.

18. Han A, Lu Y, Zheng Q, Zhang J, Zhao Y, Zhao M, Cui X. Qiliqiangxin Attenuates Cardiac Remodeling via Inhibition of TGF-beta1/Smad3 and NFkappaB Signaling Pathways in a Rat Model of Myocardial Infarction. Cell Physiol Biochem. 2018;45:1797-806

19. Zhang F, Zhang Y, Li X, Zhang S, Zhu M, Du W, Xiao X. Research on Qmarkers of Qiliqiangxin capsule for chronic heart failure treatment based on pharmacokinetics and pharmacodynamics association. Phytomedicine. 2018; 44:220-30.

20. Shen S, Jiang H, Bei Y, Zhang J, Zhang H, Zhu H, Zhang C, Yao W, Wei C, Shang $\mathrm{H}$, et al. Qiliqiangxin Attenuates Adverse Cardiac Remodeling after Myocardial Infarction in Ovariectomized Mice via Activation of PPARgamma. Cell Physiol Biochem. 2017:42:876-88.

21. Lin S, Wu X, Tao L, Bei Y, Zhang H, Zhou Y, Shen S, Xiao J, Li X. The Metabolic Effects of Traditional Chinese Medication Qiliqiangxin on H9C2 Cardiomyocytes. Cell Physiol Biochem. 2015;37:2246-56.

22. Li X, Zhang J, Huang J, Ma A, Yang J, Li W, Wu Z, Yao C, Zhang Y, Yao W, et al. A multicenter, randomized, double-blind, parallel-group, placebocontrolled study of the effects of qili qiangxin capsules in patients with chronic heart failure. J Am Coll Cardiol. 2013;62:1065-72.

23. Zhang C, Liu X, Zhang C, Li J, Guo W, Yan D, Yang C, Zhao J, Wu X, Shi J. Phosphorylated eEF2 is SUMOylated and induces cardiomyocyte apoptosis during myocardial ischemia reperfusion. J Cardiol. 2017;69:689-98.

24. He X, Li S, Liu B, Susperreguy S, Formoso K, Yao J, Kang J, Shi A, Birnbaumer $L$, Liao Y. Major contribution of the $3 / 6 / 7$ class of TRPC channels to myocardial ischemia/reperfusion and cellular hypoxia/reoxygenation injuries. Proc Natl Acad Sci U S A. 2017;114:E4582-91.

25. Benjamin EJ, Blaha MJ, Chiuve SE, Cushman M, Das SR, Deo R, de Ferranti SD, Floyd J, Fornage M, Gillespie C, et al. Heart Disease and Stroke Statistics2017 Update: A Report From the American Heart Association. Circulation. 2017;135:e146-603.

26. Davidson SM, Arjun S, Basalay MV, Bell RM, Bromage DI, Botker HE, Carr RD, Cunningham J, Ghosh AK, Heusch G, et al. The 10th Biennial Hatter Cardiovascular Institute workshop: cellular protection-evaluating new directions in the setting of myocardial infarction, ischaemic stroke, and cardio-oncology. Basic Res Cardiol. 2018;113:43.

27. Wang Y, Han X, Fu M, Wang J, Song Y, Liu Y, Zhang J, Zhou J, Ge J. Qiliqiangxin attenuates hypoxia-induced injury in primary rat cardiac microvascular endothelial cells via promoting HIF-1alpha-dependent glycolysis. J Cell Mol Med. 2018;22:2791-803.

28. Sun T, Liu H, Cheng Y, Yan L, Krittanawong C, Li S, Qian W, Su W, Chen X, Hou $X$ et al: 2,3,5,4'-Tetrahydroxystilbene-2-O-beta-d-glucoside eliminates ischemia/reperfusion injury-induced $\mathrm{H} 9 \mathrm{c} 2$ cardiomyocytes apoptosis involving in Bcl-2, Bax, caspase-3, and Akt activation. J Cell Biochem. 2019. Epub ahead of print. PMID: 30891810.

29. Botker HE, Hausenloy D, Andreadou I, Antonucci S, Boengler K, Davidson SM, Deshwal S, Devaux Y, Di Lisa F, Di Sante M, et al. Practical guidelines for rigor and reproducibility in preclinical and clinical studies on cardioprotection. Basic Res Cardiol. 2018;113:39.

30. Wang Z, Fang J, Xiao J. Correlation of the expression of inflammatory factors with expression of apoptosis-related genes Bax and $\mathrm{BCl}-2$, in burned rats. Exp Ther Med. 2019;17:1790-6.

31. Yang B, Ye D, Wang Y. Caspase-3 as a therapeutic target for heart failure. Expert Opin Ther Targets. 2013;17:255-63.

32. Shirakabe A, Ikeda Y, Sciarretta S, Zablocki DK, Sadoshima J. Aging and Autophagy in the Heart. Circ Res. 2016;118:1563-76.

33. Zhou H, Zhu P, Wang J, Zhu H, Ren J, Chen Y. Pathogenesis of cardiac ischemia reperfusion injury is associated with CK2alpha-disturbed mitochondrial homeostasis via suppression of FUNDC1-related mitophagy. Cell Death Differ. 2018;25:1080-93.

34. Lee CF, Cao Y, Tian R. Failed Power Plant Turns Into Mass Murder: New Insight on Mitochondrial Cardiomyopathy. Circ Res. 2018;122:11-3.

35. Bueno M, Lai YC, Romero Y, Brands J, St CC, Kamga C, Corey C, HerazoMaya JD, Sembrat J, Lee JS, et al. PINK1 deficiency impairs mitochondrial homeostasis and promotes lung fibrosis. J Clin Invest. 2015;125:521-38.

36. Chen M, Chen Z, Wang Y, Tan Z, Zhu C, Li Y, Han Z, Chen L, Gao R, Liu L, et al. Mitophagy receptor FUNDC1 regulates mitochondrial dynamics and mitophagy. Autophagy. 2016;12:689-702.

37. Wu W, Tian W, Hu Z, Chen G, Huang L, Li W, Zhang X, Xue P, Zhou C, Liu L, et al. ULK1 translocates to mitochondria and phosphorylates FUNDC1 to regulate mitophagy. EMBO Rep. 2014;15:566-75.

\section{Publisher's Note}

Springer Nature remains neutral with regard to jurisdictional claims in published maps and institutional affiliations.

Ready to submit your research? Choose BMC and benefit from:

- fast, convenient online submission

- thorough peer review by experienced researchers in your field

- rapid publication on acceptance

- support for research data, including large and complex data types

- gold Open Access which fosters wider collaboration and increased citations

- maximum visibility for your research: over $100 \mathrm{M}$ website views per year

At $\mathrm{BMC}$, research is always in progress.

Learn more biomedcentral.com/submissions 